\title{
Effect of aluminium oxide nanoparticles on the enzymatic activity on microorganisms of activated sludge
}

\author{
Nina Doskocz ${ }^{1, *}$, Katarzyna Affek ${ }^{1}$, and Monika Załęska-Radziwiłł ${ }^{1}$ \\ ${ }^{1}$ Warsaw University of Technology, Faculty of Building Services, Hydro and Environmental \\ Engineering, Department of Biology, Nowowiejska 20, 00-653 Warsaw, Poland
}

\begin{abstract}
The increased production and commercial use of nanoparticles (NPs), combined with a lack of regulation regarding their disposal, may result in the unwanted introduction of NPs to wastewater. Wastewater nutrient removal depends on the metabolisms of activated sludge bacteria and their related key enzymes. Therefore, the aim of this work was to determine the effect of aluminium oxide nanoparticles concentrations on the activated sludge enzymatic activity of microorganisms. Tested nanoparticles inhibition cellular respiration in TTC method in the four highest tested concentrations. Moreover, in most samples observed increase dehydrogenase activity. In this study, nano- $\mathrm{Al}_{2} \mathrm{O}_{3}$ also caused a clear stimulation of the activity of hydrolytic enzymes microorganisms of activate sludge. Effects of aluminum oxide (compound in bulk forms) on enzymatic activity were different than in the case of the nano from of $\mathrm{Al}_{2} \mathrm{O}_{3}$.
\end{abstract}

\section{Introduction}

Wastewater may contain wide variety chemical substances, which can inhibit microorganism's growth and cause various problems during biological treatment. Many harmful substances, depending on their physical and chemical properties, can be not readily biodegradable and remain toxic during the entire wastewater treatment process [1]. Examples of such compounds are nanoparticles, which are used in agriculture, industrial products and in medicine [2]. Investments for their production in 2005 amounted to about 10 billion dollars, and in the years 2011-2015 they were to reach USD 1.000 billion [3].

The intensive growth of nanotechnology triggers the increase in nanoparticle content in sewage and waste, which as a consequence makes them enter the surface waters and water intended for human consumption [4]. Literature data showed, that predicted concentrations in sludge, for engineered nanoparticles (nano- $\mathrm{TiO}_{2}$, nano-Ag, nano- $\mathrm{SiO}_{2}$, nano- $\mathrm{Au}$ ) that are being used or could be used in cosmetics and personal care products and coatings, they ranged from 0.01 to $40.7 \mathrm{mg} / \mathrm{kg}$ [5].

These data indicate, nanoparticles can have some negative influence on microorganisms in activated sludge, which is a commonly used method for pollutants removal from

${ }^{*}$ Corresponding author: nina.doskocz@.pw.edu.pl 
wastewater and depends on many biochemical reactions, which are catalysed by some key enzymes in activated sludge bacteria. However, current knowledge about these effects is limited.

The inhibition effect of the nanoparticles on microorganism's growth in activated sludge has been reported $[4,6,7]$. In the literature there are also a few reports about influence NPs on biological processes of nitrogen and phosphorus removal as well as on denitrifying bacteria and microorganisms responsible for phosphorus elimination, the so-called PAO (polyphosphate accumulating organisms). Zheng et al. (2011) showed that, nano-ZnO can induce serious trouble in self-purification of water as well as in biological wastewater treatment [8].

However, current knowledge about impacts of NPs on enzymatic activates which are the excellent indicators of activated sludge microbial function is limited. Especially, dehydrogenase activity is commonly assessed as a general indicator of the oxidative capacity of microorganisms [9-12].

Therefore, the aim of the work was to determine the effect of aluminium oxide nanoparticles concentrations on the activated sludge dehydrogenase and hydrolytic activity. The scope of the investigations covers also experiments with bulk materials (compounds of the macro-form $-\mathrm{Al}_{2} \mathrm{O}_{3}$ ).

Little information is available on the destiny, transport, and effects of nanomaterials including metal-based particles such as nanosized $\mathrm{Al}_{2} \mathrm{O}_{3}$, in the environment. Nanosized aluminum is currently being used by the military and commercial industries in many applications including coatings, thermites, and propellants. The use of aluminium oxide nanoparticles in various applications may cause a release of the oxidized form of nano$\mathrm{Al}_{2} \mathrm{O}_{3}$, into the environment. As utilization of nanomaterials is on the rise, it is increasingly important to determine their potential environmental destiny and the effects $[2,7]$.

\section{Materials and Methods}

\subsection{Chemicals}

Aluminum oxide nanoparticles (nano- $\mathrm{Al}_{2} \mathrm{O}_{3}$ ), nanopowder $<50 \mathrm{~nm}$ of specific surface of $>40 \mathrm{~m}^{2} / \mathrm{g}$, aluminum oxide of purity of $>98 \%$, were obtained from Sigma-Aldrich Company. Initial solutions of the tested compounds were prepared in deionized water. Because of the tendency of aggregate formation by the compounds, the obtained solutions of the compounds were sonicated for 30 min using an ultrasonic disintegrator of MDM-10 type $(0.4 \mathrm{~kW}$ at a frequency of $20 \mathrm{kHz})$. These solutions of nano- $\mathrm{Al}_{2} \mathrm{O}_{3}$ and $\mathrm{Al}_{2} \mathrm{O}_{3}$ were diluted in a decreasing series with the ratio of geometric progression of dilutions of $\mathrm{q}=2$ obtaining solutions in the concentration range $500-0.97 \mathrm{mg} / \mathrm{l}$.

Enzymatic activity of the microorganisms in the presence of tested compounds was determined by means of the triphenyl tetrazolium chloride (TTC) test for dehydrogenases activity and test for hydrolytic activity - fluorescein diacetate (FDA) determination. The TTC test was performed in accordance with the Polish Standard PN-C-04616-8 2008. Specific enzyme activity was estimated in a spectrophotometer at $490 \mathrm{~nm}$ by measuring the concentration of triphenyl formazan (TF) formed from TTC reduction.

Similarly, The TTC calibration curve was made as follows: introduced into tubes respectively $0.1 ; 0.2 ; 0,3,0.4 ; 0.5 \mathrm{ml}$ of $\mathrm{TF}$ and made up to $5 \mathrm{ml}$ with butanol. The obtained standard solutions contain respectively: $2.0 ; 4.0 ; 6.0 ; 8.0$ and $10 \mu \mathrm{mol} \mathrm{TF}$ in a $5 \mathrm{ml}$ sample. Absorbance measurement of the above solutions using a spectrophotometer at $490 \mathrm{~nm}$. 
Determination of TTC dehydrogenase activity was made as follows: introduced into tubes a suspension of bacterial cells in a volume of $5 \mathrm{ml}$. The cell suspension was prepared by inoculating $100 \mathrm{ml}$ of broth medium with $0,5 \mathrm{ml}$ of activated sludge after incubation with test compounds after $30 \mathrm{~min}$. and $24 \mathrm{~h}$. The cultures were incubated at $26^{\circ} \mathrm{C}$ in $48 \mathrm{~h}$. After incubation, the cultures were centrifuged and suspended in buffered water. $7 \mathrm{~mL}$ Tris$\mathrm{HCl}$ buffer, $1 \mathrm{ml} \mathrm{Na} \mathrm{SO}_{3}(0.36 \%)$ were added into each tube. Incubation was carried out at $37^{\circ} \mathrm{C}$ under shaking conditions $(120 \mathrm{rpm})$ in the dark for $30 \mathrm{~min} .2 \mathrm{ml}$ sulfuric acid was added to terminate enzyme reaction. $10 \mathrm{ml}$ of butanol was added to the samples, mixed thoroughly and placed in a water bath with a temperature of $90^{\circ} \mathrm{C}$ for $5 \mathrm{~min}$. Then $5 \mathrm{ml}$ of butanol extract from each tube were centrifuged at $6000 \mathrm{rpm}$ for $5 \mathrm{~min}$. The TF was measured at $490 \mathrm{~nm}$ in the spectrophotometer. The results were given in $\mu \mathrm{mol}$ of TF per $\mathrm{s}$ and $\mathrm{kg}$ of dry weight (d.w.) [13].

The FDA test was performed in accordance with the Schnürer and Rosswall method [14]. The bacterial suspensions in the phosphate buffer ( $\mathrm{pH} 7.6$ ) with FDA in acetone $\left(20 \mathrm{mg} \mathrm{cm}^{-3}\right.$ ) were incubated at $26^{\circ} \mathrm{C}$ under shaking conditions. The enzymatic reaction was stopped by the addition of acetone in a volume ratio of 1:1. The concentration of produced fluorescein was determined spectrophotometrically at $490 \mathrm{~nm}$. The enzymatic activity is reported as $\mathrm{mg}$ of fluorescein $\mathrm{s}^{-1} \mathrm{~g}^{-1} \mathrm{~d} . \mathrm{w}$. [15].

To perform TTC and FDA tests the appropriate chemicals and activated sludge working in laboratory conditions, in Department of Biology, Faculty of Building Services, Hydro and Environmental Engineering, Warsaw University of Technology were used.

Assessment of inhibition activity of the hydrolase was made after $30 \mathrm{~min}$ and 24 hours of activated sludge incubation with the tested compounds. Each experiment was done in 3 replicates. The mean values and standard deviations was then calculated for each experimental group exposed to tested compounds. Samples containing activated sludge that had not previously been treated with the test compounds were used as control. To clear the presentation of the results, the enzyme activity data of treated activated sludge samples were expressed as the percentage of the enzyme activity compared to the control sample.

Inhibition of enzymatic activity was calculated according to Eq. 1.

$$
I=\frac{B_{c}-B_{n}}{B_{c}} \cdot 100[\%]
$$

$I_{c}$ - Inhibition of enzymatic activity

$B_{c}$ - Optical density of suspension in control sample after time $\mathrm{t}$

$B_{n}-$ Optical density of suspension in the sample examined after time $\mathrm{t}$

\section{Results and discussion}

The enzymatic activities in activated sludge treated with two different compounds: aluminium oxide NPs, and their bulk form $-\mathrm{Al}_{2} \mathrm{O}_{3}$ were measured after 30 min and $24 \mathrm{~h}$ of exposure. In enzymatic tests (TTC and FDA tests) with microorganisms from activated sludge, were found inhibitions and stimulation of enzyme activity.

The dehydrogenase activity was changing rapidly. The activity in samples with nano- $\mathrm{Al}_{2} \mathrm{O}_{3}$ after 30 minutes ranged from 42.2 to almost $337.4 \mu \mathrm{mol} \mathrm{TF} / \mathrm{kg}$ d.w $\mathrm{s}^{-1}$ and increased with decreasing in the concentration of the tested NPs. After 24 hours observed increased of dehydrogenase activity with increasing concentration of tested NPs - from 82.2 to almost $192.4 \mu \mathrm{mol} \mathrm{TF} / \mathrm{kg}$ d.w. $\mathrm{s}^{-1}$ (table 1).

Aluminum oxide nanoparticles, after 30 minutes contact with microorganisms in concentrations from $500-31 \mathrm{mg} / \mathrm{l}$, inhibited the activity of dehydrogenases from $66 \%$ 
to $0.39 \%$, respectively. At lower concentrations NPs clearly, stimulated activity of this enzyme.

After $24 \mathrm{~h}$, in concentrations of $500-15 \mathrm{mg} / \mathrm{L}$, stimulation of dehydrogenase activity was also found, while in the lower concentration, inhibition of enzymatic activity was observed (figure 1).

Hydrolytic activity was slowly decreasing from the beginning till the end of the duration of the test (table 1). Nano- $\mathrm{Al}_{2} \mathrm{O}_{3}$ regardless of the time of action on activated sludge microorganisms caused a clear stimulation of the activity of hydrolytic enzymes in these microorganisms (figure 3).

The obtained results also show that the tested nanoparticle a different impact on dehydrogenase and hydrolytic activities than the same compound in the bulk form (table 2), (figure 2 and 4). Aluminium oxide greater stimulated dehydrogenase activity and to a lesser extent stimulated hydrolytic activity than compound in nano form.

Table 1. Dehydrogenase and hydrolytic activity in the presence of nano- $\mathrm{Al}_{2} \mathrm{O}_{3}$.

\begin{tabular}{|c|c|c|c|c|c|}
\hline \multirow{2}{*}{ 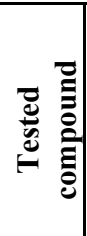 } & \multirow{2}{*}{ 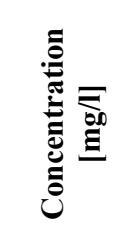 } & \multicolumn{2}{|c|}{ 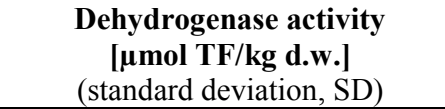 } & \multicolumn{2}{|c|}{$\begin{array}{c}\text { Hydrolytic activity } \\
{\left[\boldsymbol{\mu g} \text { fluorescein/kg of d.w. } \mathbf{s}^{-1} \text { ] }\right.} \\
\text { (standard deviation, SD) }\end{array}$} \\
\hline & & after $30 \mathrm{~min}$ & after $24 \mathrm{~h}$ & after $30 \mathrm{~min}$ & after $24 \mathrm{~h}$ \\
\hline \multirow{11}{*}{ 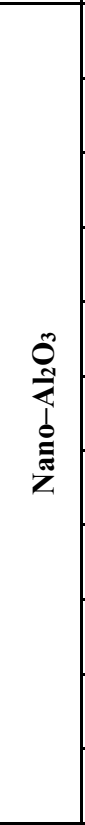 } & 500 & $\begin{array}{c}42.2 \\
(42.7-42.5)\end{array}$ & $\begin{array}{c}192.4 \\
(192.2-193.0)\end{array}$ & $\begin{array}{c}704.0 \\
(74.9-704.2)\end{array}$ & $\begin{array}{c}16.6 \\
(16.3-16.1)\end{array}$ \\
\hline & 250 & $\begin{array}{c}82.4 \\
(82.5-81.7) \\
\end{array}$ & $\begin{array}{c}193.1 \\
(193.6-194.2)\end{array}$ & $\begin{array}{c}406.9 \\
(406.4-406.5)\end{array}$ & $\begin{array}{c}15.9 \\
(15.1-16.7) \\
\end{array}$ \\
\hline & 125 & $\begin{array}{c}88.5 \\
(88.8-89.1) \\
\end{array}$ & $\begin{array}{c}161.8 \\
(161.5-161.4)\end{array}$ & $\begin{array}{c}384.7 \\
(384.9-384.1)\end{array}$ & $\begin{array}{c}15.8 \\
(15.8-15.1) \\
\end{array}$ \\
\hline & 62.5 & $\begin{array}{c}118.8 \\
(118.6-119.5)\end{array}$ & $\begin{array}{c}135.0 \\
(135.0-136.4)\end{array}$ & $\begin{array}{c}357.6 \\
(358.2-357.8)\end{array}$ & $\begin{array}{c}11.2 \\
(11.4-11.5)\end{array}$ \\
\hline & 31.3 & $\begin{array}{c}123.9 \\
(123.7-122.9)\end{array}$ & $\begin{array}{c}126.1 \\
(125.7-126.9)\end{array}$ & $\begin{array}{c}319.4 \\
(319.9-320.8)\end{array}$ & $\begin{array}{c}10.9 \\
(11.1-10.7)\end{array}$ \\
\hline & 15.6 & $\begin{array}{c}138.1 \\
(138.1-138.6) \\
\end{array}$ & $\begin{array}{c}115.9 \\
(115.9-116.3) \\
\end{array}$ & $\begin{array}{c}188.9 \\
(188.6-188.9) \\
\end{array}$ & $\begin{array}{c}10.6 \\
(10.6-10.7) \\
\end{array}$ \\
\hline & 7.8 & $\begin{array}{c}195.0 \\
(195.2-196.0) \\
\end{array}$ & $\begin{array}{c}110.6 \\
(123.7-122.9) \\
\end{array}$ & 155.7 & $\begin{array}{c}10.4 \\
(10.3-10.3) \\
\end{array}$ \\
\hline & 3.9 & $\begin{array}{c}203.4 \\
(203.9-203.4)\end{array}$ & $\begin{array}{c}98.7 \\
(98.8-98.7) \\
\end{array}$ & $\begin{array}{c}117.8 \\
(117.7-118.9)\end{array}$ & $\begin{array}{c}8.8 \\
(8.6-8.4)\end{array}$ \\
\hline & 1.9 & $\begin{array}{c}231.4 \\
(231.7-232.3)\end{array}$ & $\begin{array}{c}96.7 \\
(96.3-96.3) \\
\end{array}$ & $\begin{array}{c}74.3 \\
(73.9-74.5) \\
\end{array}$ & $\begin{array}{c}8.6 \\
(8.7-9.1)\end{array}$ \\
\hline & 0.97 & $\begin{array}{c}337.4 \\
(338.3-337.8)\end{array}$ & $\begin{array}{c}82.2 \\
(82.8-81.7)\end{array}$ & $\begin{array}{c}69.2 \\
(69.4-68.7) \\
\end{array}$ & $\begin{array}{c}4.3 \\
(4.2-4.7)\end{array}$ \\
\hline & Control & $\begin{array}{c}124.4 \\
(124.7-124.3)\end{array}$ & $\begin{array}{c}110.8 \\
(110.8-111.1)\end{array}$ & $\begin{array}{c}19.6 \\
(19.8-19.3)\end{array}$ & $\begin{array}{c}0.7 \\
(0.6-0.7)\end{array}$ \\
\hline
\end{tabular}


Table 2. Dehydrogenase and hydrolytic activity in the presence of $\mathrm{Al}_{2} \mathrm{O}_{3}$.

\begin{tabular}{|c|c|c|c|c|c|}
\hline \multirow{2}{*}{ 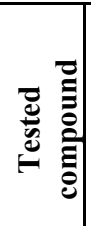 } & \multirow{2}{*}{ 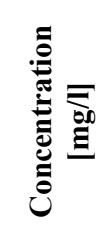 } & \multicolumn{2}{|c|}{$\begin{array}{c}\text { Dehydrogenase activity } \\
\text { [ } \mu \mathrm{mol} \text { TF/kg d.w.] } \\
\text { (standard deviation, SD) }\end{array}$} & \multicolumn{2}{|c|}{$\begin{array}{c}\text { Hydrolytic activity } \\
{\left[\boldsymbol{\mu g} \text { fluorescein/kg of d.w. } \mathbf{s}^{-1} \text { ] }\right.} \\
\text { (standard deviation, SD) }\end{array}$} \\
\hline & & after $30 \mathrm{~min}$ & after $24 \mathrm{~h}$ & after $30 \mathrm{~min}$ & after $24 \mathrm{~h}$ \\
\hline \multirow{11}{*}{ 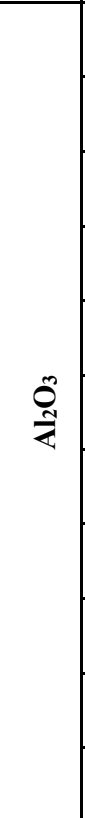 } & 500 & $\begin{array}{c}68.2 \\
(68.7-69.5)\end{array}$ & $\begin{array}{c}265.8 \\
(265.9-265.8)\end{array}$ & $\begin{array}{c}744.3 \\
(744.9-745.1)\end{array}$ & $\begin{array}{c}20.5 \\
(20.3-20.9)\end{array}$ \\
\hline & 250 & $\begin{array}{c}72.5 \\
(73.4-72.9) \\
\end{array}$ & $\begin{array}{c}239.8 \\
(239.7-239.3)\end{array}$ & $\begin{array}{c}542.8 \\
(5455.1-541.9)\end{array}$ & $\begin{array}{c}19.6 \\
(19.3-20.1)\end{array}$ \\
\hline & 125 & $\begin{array}{c}98.5 \\
(98.8-99.7)\end{array}$ & $\begin{array}{c}211.8 \\
(211.1-212.2)\end{array}$ & $\begin{array}{c}486.2 \\
(485.2-485.8)\end{array}$ & $\begin{array}{c}18.6 \\
(20.3-20.4)\end{array}$ \\
\hline & 62.5 & $\begin{array}{c}120.9 \\
(120.1-120.9)\end{array}$ & $\begin{array}{c}208.4 \\
(207.9-210.4)\end{array}$ & $\begin{array}{c}457.4 \\
(457.9-457.8)\end{array}$ & $\begin{array}{c}17.0 \\
(17.8-18.4)\end{array}$ \\
\hline & 31.3 & $\begin{array}{c}178.3 \\
(178.2-179.6) \\
\end{array}$ & $\begin{array}{c}206.9 \\
(204.9-206.6) \\
\end{array}$ & $\begin{array}{c}419.2 \\
(419.6-420.1) \\
\end{array}$ & $\begin{array}{c}16.0 \\
(16.5-16.5) \\
\end{array}$ \\
\hline & 15.6 & $\begin{array}{c}209.3 \\
(208,9-209.2) \\
\end{array}$ & $\begin{array}{c}195.7 \\
(214.9-212.5) \\
\end{array}$ & $\begin{array}{c}378.6 \\
(265.9-265.8) \\
\end{array}$ & $\begin{array}{c}14.3 \\
(14.8-15.1) \\
\end{array}$ \\
\hline & 7.8 & $\begin{array}{c}213.7 \\
(214.9-212.8) \\
\end{array}$ & $\begin{array}{c}171.7 \\
(172.5-171.8) \\
\end{array}$ & $\begin{array}{c}275.3 \\
(274.9-275.5) \\
\end{array}$ & $\begin{array}{c}11.7 \\
(11.6-11.7) \\
\end{array}$ \\
\hline & 3.9 & $\begin{array}{c}241.7 \\
(241.8-242.1) \\
\end{array}$ & $\begin{array}{c}153.4 \\
(153.2-153.7) \\
\end{array}$ & $\begin{array}{c}217.8 \\
(218.4-217.2) \\
\end{array}$ & $\begin{array}{c}10.4 \\
(17.8-18.4) \\
\end{array}$ \\
\hline & 1.9 & $\begin{array}{c}271.8 \\
(272.8-270.9) \\
\end{array}$ & $\begin{array}{c}137.8 \\
(214.9-212.8) \\
\end{array}$ & $\begin{array}{c}104.3 \\
(104.9-104.9) \\
\end{array}$ & $\begin{array}{c}9.8 \\
(9.1-8.8) \\
\end{array}$ \\
\hline & 0.97 & $\begin{array}{c}367.2 \\
(366.8-367.1) \\
\end{array}$ & $\begin{array}{c}92.2 \\
(92.1-93.6) \\
\end{array}$ & $\begin{array}{c}85.1 \\
(82.8-85.6) \\
\end{array}$ & $\begin{array}{c}6.3 \\
(6.2-6.6) \\
\end{array}$ \\
\hline & Control & $\begin{array}{c}125.8 \\
(126.1-125.4)\end{array}$ & $\begin{array}{c}115.6 \\
(115.4-115.2)\end{array}$ & $\begin{array}{c}20.1 \\
(20.3-20.4)\end{array}$ & $\begin{array}{c}1.6 \\
(1.7-1.5)\end{array}$ \\
\hline
\end{tabular}

Table 3. Percentage of inhibition and stimulation of dehydrogenase activity in the presence of nano- $\mathrm{Al}_{2} \mathrm{O}_{3}$ and $\mathrm{Al}_{2} \mathrm{O}_{3}$.

\begin{tabular}{|c|c|c|c|}
\hline \multirow{2}{*}{ 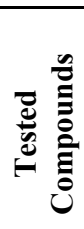 } & \multirow{2}{*}{ 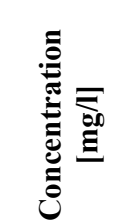 } & \multicolumn{2}{|c|}{$\begin{array}{c}\text { Percentage of inhibition and stimulation of } \\
\text { dehydrogenase activity [\%] (standard } \\
\text { deviation, SD) }\end{array}$} \\
\hline & & after $30 \mathrm{~min}$ & after $24 \mathrm{~h}$ \\
\hline \multirow{8}{*}{ 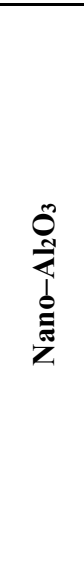 } & 500 & $\begin{array}{c}66.07 \\
(64.87-59.75) \\
\end{array}$ & $\begin{array}{c}-78.19 \\
(-76.72-(-78.46) \\
\end{array}$ \\
\hline & 250 & $\begin{array}{c}33.72 \\
(29.69,87-34.55) \\
\end{array}$ & $\begin{array}{c}-74.32 \\
(-75.35-(-74.61)\end{array}$ \\
\hline & 125 & $\begin{array}{c}28.82 \\
(29.71-31.89)\end{array}$ & $\begin{array}{c}-46.01 \\
(-46.23-(-45.33)\end{array}$ \\
\hline & 62.5 & $\begin{array}{c}4.50 \\
(4.22-6.43)\end{array}$ & $\begin{array}{c}-21.89 \\
(-46.23-(-45.33)\end{array}$ \\
\hline & 31.3 & $\begin{array}{c}0.39 \\
(0,76-0,38)\end{array}$ & $\begin{array}{c}-13.80 \\
(-12.97-(-13.84)\end{array}$ \\
\hline & 15.6 & $\begin{array}{c}-11.04 \\
(-14.39-(-16.37)\end{array}$ & $\begin{array}{c}-4.65 \\
(-4.23-(-4.57)\end{array}$ \\
\hline & 7.8 & $\begin{array}{c}-56.8 \\
(-49.92-(-56.74)\end{array}$ & $\begin{array}{c}0.13 \\
(-4.23-(-4.57)\end{array}$ \\
\hline & 3.9 & -63.53 & 10.86 \\
\hline
\end{tabular}




\begin{tabular}{|c|c|c|c|}
\hline & & $(-64.37-(-63.25)$ & $(-10.54-(-10.29)$ \\
\hline & 1.9 & $\begin{array}{c}-86.02 \\
(-86.72-(-86.46)\end{array}$ & $\begin{array}{c}12.71 \\
(-12.66-(-13.01)\end{array}$ \\
\hline & 0.97 & $\begin{array}{c}-171.24 \\
(-172.36-(-170.63)\end{array}$ & $\begin{array}{c}25.77 \\
(-4.23-(-4.57)\end{array}$ \\
\hline & Control & 0.00 & 0.00 \\
\hline \multirow{11}{*}{ 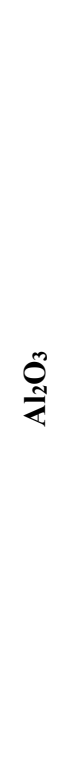 } & 500 & $\begin{array}{c}45.80 \\
(45.71-34.79)\end{array}$ & $\begin{array}{c}-129.82 \\
(-128.92-(-129.58)\end{array}$ \\
\hline & 250 & $\begin{array}{c}42.37 \\
(41.63-42.09)\end{array}$ & $\begin{array}{c}-107.37 \\
(-104.76-(-107.81)\end{array}$ \\
\hline & 125 & $\begin{array}{c}21.70 \\
(21.87-22.32)\end{array}$ & $\begin{array}{c}-83.14 \\
(-84.03-(-83.63)\end{array}$ \\
\hline & 62.5 & $\begin{array}{c}3.95 \\
(2.79-3.61)\end{array}$ & $\begin{array}{c}-80.26 \\
(-80.25-(-79.83)\end{array}$ \\
\hline & 31.3 & $\begin{array}{c}-41.67 \\
(-41.94-(-41.75)\end{array}$ & $\begin{array}{c}-78.93 \\
(-75.35-(-74.61)\end{array}$ \\
\hline & 15.6 & $\begin{array}{c}-66.3 \\
(-66.75-(-66.74)\end{array}$ & $\begin{array}{c}-69.28 \\
(-68.37-(-69.03)\end{array}$ \\
\hline & 7.8 & $\begin{array}{c}-69.75 \\
(-69.72-(-70.40) \\
\end{array}$ & $\begin{array}{c}-48.44 \\
(-48.62-(-48.57) \\
\end{array}$ \\
\hline & 3.9 & $\begin{array}{c}-92.04 \\
(-92.86-(-91.39) \\
\end{array}$ & $\begin{array}{c}-32.63 \\
(-31.99-(-32.53) \\
\end{array}$ \\
\hline & 1.9 & $\begin{array}{c}-115.95 \\
(-114.72-(-115.58)\end{array}$ & $\begin{array}{c}-19.19 \\
(-41.94-(-41.75) \\
\end{array}$ \\
\hline & 0.97 & $\begin{array}{c}-191.72 \\
(-192.37-(-191.55)\end{array}$ & $\begin{array}{c}14.19 \\
(-14.66-(-14.37) \\
\end{array}$ \\
\hline & Control & 0.00 & 0.00 \\
\hline
\end{tabular}

Table 4. Percentage of inhibition and stimulation of hydrolytic activity in the presence of nano- $\mathrm{Al}_{2} \mathrm{O}_{3}$ and $\mathrm{Al}_{2} \mathrm{O}_{3}$.

\begin{tabular}{|c|c|c|c|}
\hline \multirow{2}{*}{ 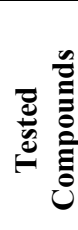 } & \multirow{2}{*}{ 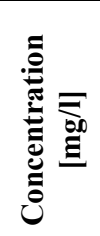 } & \multicolumn{2}{|c|}{$\begin{array}{c}\text { Percentage of inhibition and stimulation } \\
\text { of hydrolytic activity } \\
\text { [\%] (standard deviation, SD) } \\
\end{array}$} \\
\hline & & after $30 \mathrm{~min}$ & after $24 \mathrm{~h}$ \\
\hline \multirow{9}{*}{ 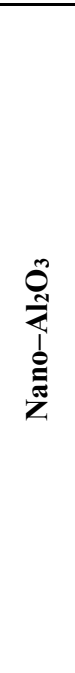 } & 500 & $\begin{array}{c}-3489.94 \\
(-3488.92-(-3529.51)\end{array}$ & $\begin{array}{c}-2339.63 \\
(-2338.34-(-2327.56) \\
\end{array}$ \\
\hline & 250 & $\begin{array}{c}-1974.90 \\
(-1976.33-(-1965.75)\end{array}$ & $\begin{array}{c}-2248.21 \\
(-2234.45-(-2248.21) \\
\end{array}$ \\
\hline & 125 & $\begin{array}{c}-1861.55 \\
(-1931.91-(-1868.73)\end{array}$ & $\begin{array}{c}-2228.63 \\
(-2233.07-(-2246.45)\end{array}$ \\
\hline & 62.5 & $\begin{array}{c}-1723.27 \\
(-1753.66-(-1739.38)\end{array}$ & $\begin{array}{c}-1545.75 \\
(-1544.54-(-1574.22)\end{array}$ \\
\hline & 31.3 & $\begin{array}{c}-1528.73 \\
(-1531.78-(-1561.44) \\
\end{array}$ & $\begin{array}{c}-1517.99 \\
(-1538.67-(-1527.51) \\
\end{array}$ \\
\hline & 15.6 & $\begin{array}{c}-863.05 \\
(-864.49-(-863.55) \\
\end{array}$ & $\begin{array}{c}-1461.89 \\
(-1437.78-(-1483.98) \\
\end{array}$ \\
\hline & 7.8 & $\begin{array}{c}-693.85 \\
(-691.40-(-694.02)\end{array}$ & $\begin{array}{c}-1438.83 \\
(-1421.73-(-1481.56) \\
(-10\end{array}$ \\
\hline & 3.9 & $\begin{array}{c}-500.76 \\
(-501.41-(-500.41)\end{array}$ & $\begin{array}{c}-1202.13 \\
(-1266.12-(-12,34.16) \\
\end{array}$ \\
\hline & 1.9 & $\begin{array}{c}-278.85 \\
(-280.64-(-277.57)\end{array}$ & $\begin{array}{c}-1162.73 \\
(-1144.54-(-1176.69)\end{array}$ \\
\hline
\end{tabular}




\begin{tabular}{|c|c|c|c|}
\hline & 0.97 & $\begin{array}{c}-252.88 \\
(-251.19-(-252.27)\end{array}$ & $\begin{array}{c}-542.33 \\
(-541.46-(-543.22)\end{array}$ \\
\hline & Control & 0.00 & 0.00 \\
\hline \multirow{11}{*}{ 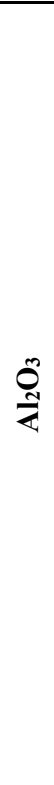 } & 500 & $\begin{array}{c}-3595.78 \\
(-3596.44-(-3595.84) \\
\end{array}$ & $\begin{array}{c}-1052.72 \\
(-1052.67-(-1053.17)\end{array}$ \\
\hline & 250 & $\begin{array}{c}-2595.13 \\
(-2595.85-(-2595.26)\end{array}$ & $\begin{array}{c}-999.89 \\
(-998.76-(-999.89)\end{array}$ \\
\hline & 125 & $\begin{array}{c}-2313.95 \\
(-2312.39-(-2327.56)\end{array}$ & $\begin{array}{c}-943.13 \\
(-944.77-(-941.65)\end{array}$ \\
\hline & 62.5 & $\begin{array}{c}-2171.25 \\
(-2171.68-(-2171.77)\end{array}$ & $\begin{array}{c}-856.58 \\
(-956.13-(-855.880\end{array}$ \\
\hline & 31.3 & $\begin{array}{c}-1981.58 \\
(-1982.93-(-1982.43)\end{array}$ & $\begin{array}{c}-801.50 \\
(-801.27-(-802.06)\end{array}$ \\
\hline & 15.6 & $\begin{array}{c}-1779.64 \\
(-1779.54-(-1779.22)\end{array}$ & $\begin{array}{c}-705.95 \\
(-705.74-(-706.28)\end{array}$ \\
\hline & 7.8 & $\begin{array}{c}-1266.88 \\
(-1254.55-(-1274.45) \\
\end{array}$ & $\begin{array}{c}-558.14 \\
(-558.53-(-558.87) \\
\end{array}$ \\
\hline & 3.9 & $\begin{array}{c}-981.28 \\
(-984.76-(-981.89) \\
\end{array}$ & $\begin{array}{c}-483.95 \\
(-483.04-(-482.74) \\
\end{array}$ \\
\hline & 1.9 & $\begin{array}{c}-418.12 \\
(-418.27-(-429.06) \\
\end{array}$ & $\begin{array}{c}-449.67 \\
(-449.64-(-449.57) \\
\end{array}$ \\
\hline & 0.97 & $\begin{array}{c}-322.79 \\
(-324.04-(-321.94) \\
\end{array}$ & $\begin{array}{c}-256.33 \\
(-256.58-(-256.39) \\
\end{array}$ \\
\hline & Control & 0.00 & 0.00 \\
\hline
\end{tabular}

Rapidly developing industry raises concerns about the environmental impacts of nanoparticles, but the effects of nanoparticles on bacterial metabolism in wastewater treatment remain unclear. The comparison of dehydrogenases' and hydrolytic activity between samples with activated sludge containing nano- $\mathrm{Al}_{2} \mathrm{O}_{3}$ and the control samples (activated sludge without NPs) proved that tested nanoparticles can influence on metabolism of the bacteria in the suspension. After $24 \mathrm{~h}$ in high tested concentration, nano- $\mathrm{Al}_{2} \mathrm{O}_{3}$ caused decrease in dehydrogenase activity, suggesting biocidal properties of these nanoparticles. In most samples we observed increase enzyme activities. This may be related with the role of potentially released $\mathrm{Al}$ ions which are a part of the active site of the enzyme [16]. Therefore, the presence of Al can have a positive effect on the activity of that enzyme [17]. Ions released from the NPs can only partially be responsible for the toxic or stimulated effect NPs. Stimulation of enzymatic activity of microorganisms in activated sludge may be disadvantageous. May lead to an increased demand for oxygen in biomass biodegradation process and can lead to the formation of anaerobic zones. These results may suggest that the presence of nanoparticles might negatively influence the communities of micro-organisms participating in biological processes.

The data found in literary sources about effect of nanoparticles on enzymatic activity, confirm our studies. Chojniak et al. (2017) also not observed decrease in respiratory activity, moreover confirmed activity of silver NPs as anti-biofilm agent [18].

In turn, study of Asadishad et al. (2017) showed, that the nanoparticles (citrate-Gold $50 \mathrm{~nm}$, PVP-Gold $50 \mathrm{~nm}$, PVP-Gold $5 \mathrm{~nm}$, PVP-Gold $100 \mathrm{~nm}$ ) caused an initial decrease in enzyme activity after 2 hours of exposure. We also observed (after $24 \mathrm{~h}$ in high tested concentration) inhibition cellular respiration in TTC method [19].

Furthermore, our previous study [7] on NPs' influence on planktonic and biofilm bacteria participate in wastewater treatment, showed inhibitory effects of $\mathrm{Al}_{2} \mathrm{O}_{3}$ NPs in higher concentrations and stimulation of the bacteria growth in lower concentrations, which confirms that the tested NPS in this study (after $24 \mathrm{~h}$ at high concentrations), can 
inhibit growth/amount of microorganisms of activated sludge, and thus, affect their metabolism and wastewater treatment processes.

The obtained results shown that the effects of nano- $\mathrm{Al}_{2} \mathrm{O}_{3}$ on bacteria and their metabolism were stronger/different than those observed for their bulk counterparts (table 2, figure 2 and 4).

NPs have greater specific surface area, and therefore greater reactivity and potential for generating ROS (reactive oxygen species) than their bulk counterparts, and thus their expected inhibition or stimulation of activity should be greater, however, the opposite tendency can also be observed. In our study we observed greater inhibition after $30 \mathrm{~min}$ and smaller stimulation after $24 \mathrm{~h}$ of dehydrogenase activity in the presence of the nano- than the bulk form of $\mathrm{Al}_{2} \mathrm{O}_{3}$. In the case of hydrolytic activity, $\mathrm{Al}_{2} \mathrm{O}_{3}$ caused greater less stimulation the nano form of $\mathrm{Al}_{2} \mathrm{O}_{3}$. Lower toxic effects for nano from in some samples may have been determined by their tendency to aggregation and solubility. Aggregation causes a decrease of specific surface area of nanoparticles and of their solubility, and hence, lowers reactivity $[19,20]$. Similar conclusions were obtained by Kim et al. (2011) who also observed different dehydrogenase activity in the presence of the nano- than the bulk form of $\mathrm{CuO}$ [9].

\section{Conclusions}

The conducted studies concerning the impact of aluminum oxide nanoparticles (nano- $\mathrm{Al}_{2} \mathrm{O}_{3}$ ) on the enzymatic activity on microorganisms of activated sludge allowed formulating the following conclusions:

- Aluminum oxides nanoparticles caused decrease in dehydrogenase activity after 2 hours of exposure;

- Tested nanoparticles inhibition cellular respiration in TTC method only in the four highest tested concentrations;

- Nano- $\mathrm{Al}_{2} \mathrm{O}_{3}$ caused at high concentrations caused a clear stimulation of dehydrogenase activity after 30 minutes as well as after 24 hours in low concentrations;

- Nano- $\mathrm{Al}_{2} \mathrm{O}_{3}$ caused a clear stimulation of the activity of hydrolytic enzymes in microorganisms of activate sludge;

- Effects of aluminum oxide $\left(\mathrm{Al}_{2} \mathrm{O}_{3}\right)$ on enzyme activity were different than in the case of the nano forms.

This research confirmed the data found in literary sources and showed that the presence of nanoparticles might influence on biological processes of wastewater treatment by negative effects on enzyme activity of microorganisms participating in this process

It was found that nano forms of the tested compounds were posing different risks to microorganisms involved in biological wastewater treatment than the same compounds in the bulk form. Therefore, available ecotoxicity data about these compounds cannot be used to assess the harmfulness of their nano form counterparts.

This study increased the ecotoxicological knowledge and database in relation to the effect of aluminum oxide nanoparticles on enzymes in activated sludge bacteria, which catalyze many biochemical reactions, involved in to pollutants removal from wastewater. Due to specific features which are characteristic for nanocompounds, it is also important to study the interaction mechanism of nanoparticles with microbial cells on molecular level. 


\section{References}

1. A. Makuch, K. Medrzycka, E. Płaza, Research and application of new technologies in wastewater treatment and municipal solid waste disposal in Ukraine, Sweden and Poland: Proceedings of a Polish-Swedish-Ukrainian seminar / [ed] E. Plaza, E. Levlin, 113-122 (2010)

2. M. Załęska-Radziwiłł, N. Doskocz, Desalination Water Treat., 57, 1573-1581 (2016)

3. E. Navarro, A. Baun, R. Behra, N.B. Hartmann, J. Filser, A.J. Miao, A. Quigg, P.H. Santschi, L. Sigg, Ecotoxicology 17, 372-386 (2008)

4. Z. Sheng, Y. Liu, Water Res. 45, 6039-6050 (2011)

5. A. Boxall, Q. Chaudhry, A. Jones, B. Jefferson, C. Watts, Current and future predicted environmental exposure to engineered nanoparticles (Central Science Laboratory, York, 2007)

6. O. Choi, Effect of silver nanoparticles on planktonic and biofilm cell growth (A Dissertation presented to the Faculty of the Graduate School at the University of Missouri-Columbia, Columbia, 2008)

7. N. Chrzanowska, M. Załęska-Radziwiłł, Desalination Water Treat. 52, 3680-3689 (2014)

8. X. Zheng, R. Wu, Y. Chen, Environ. Sci. Technol. 45, 2826-2832 (2011)

9. S. Kim, J. Kim, I. Lee, Chem. Ecol. 27, 49-55 (2011)

10. W. Shi, J. Becker, M. Bischoff, R.F. Turco, A.E. Konopka, App. Environ. Microbiol. 68, 3859-3866 (2002)

11. R.P. Dick, D.P. Breakwell, R.F. Turco, Methods for Assessing Soil Quality, Soil Science Society American (Madison, WI, 247-271 1996)

12. L.V. Verchot, T. Borelli, Biol. Biochem. 37, 625-633 (2005)

13. PN-C-04616-8 - Water and sewage - Special investigations of sludge - Part 8: Determination of dehydrogenase activity in activated sludge by spectrophotometric method with triphenyltetrazolium chloride (2008)

14. J. Schnürer, J. Rosswall, Appl. Environ. Microbiol. 43, 1256-1261 (1982)

15. A. Tabernacka, E. Zborowska, K. Pogoda, M. Żołądek, Environ. Technol. (2017)

16. J.C. Polacco, P. Mazzafera, T. Tezotto, Plant Sci. 199-200, 79-90 (2013)

17. D.A. Dalton, H.J. Evans, F.J. Hanus, Plant Soil 88, 245-258 (1985)

18. J. Chojniak, I. Biedroń, G. Płaza, E3S Web of Conferences 17, 1-8 (2017)

19. B. Asadishad, S. Chahal, V. Cianciarelli, K. Zhou, N. Tufenkji, Environ. Sci-Nano. 4, 907-918 (2017)

20. R. Dinesh, M. Anandaraj, V. Srinivasan, S. Hamza, Geoderma 173-174, 19-27 (2012)

21. J.H. Priester, Y. Ge, R.E. Mielke, A.M. Horst, S.C. Moritz, K. Espinosa, J. Gelb, S.L. Walker, R.M. Nisbet, Y.-J. An, J.P. Schimel, R.G. Palmer, J.A. HernandezViezcas, L. Zhao, J.L. Gardea-Torresdey, P.A. Holden, Proc. Natl. Acad. Sci. 109, 2451-2456 (2012) 